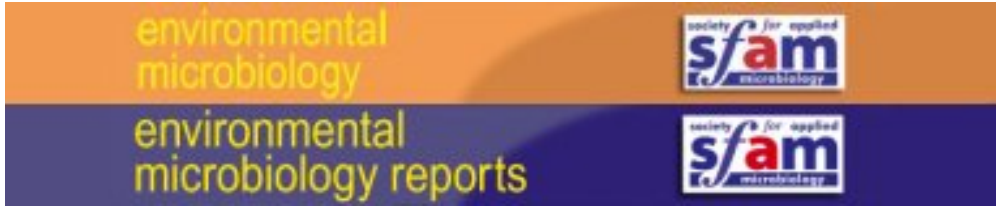

\title{
Crosstalk between bacterial conjugation and motility is mediated by plasmid-borne regulators
}

\begin{tabular}{|r|l|}
\hline Journal: & Environmental Microbiology and Environmental Microbiology Reports \\
\hline Manuscript ID & Draft \\
\hline Journal: & Environmental Microbiology Reports \\
\hline Manuscript Type: & EMIR - Brief report \\
\hline Complete List of Authors: & n/a \\
& $\begin{array}{l}\text { Luque, Ainara; University of Barcelona, Genetics, Microbiology and } \\
\text { Statistics } \\
\text { Paytubi, Sonia; University of Barcelona, Genetics, Microbiology and } \\
\text { Statistics } \\
\text { Sánchez, Javier; University of Barcelona, Genetics, Microbiology and } \\
\text { Statistics } \\
\text { Gibert, Marta; University of Barcelona, Genetics, Microbiology and } \\
\text { Statistics } \\
\text { Balsalobre, Carlos; University of Barcelona, Genetics, Microbiology and } \\
\text { Statistics (School of Biology) } \\
\text { Madrid Xufré, Cristina; University of Barcelona, Genetics, Microbiology } \\
\text { and Statistics }\end{array}$ \\
\hline Keywords: & R27, conjugation, motility, TrhR TrhY, HtdA \\
\hline
\end{tabular}

\section{SCHOLARONE" Manuscripts}


1 Crosstalk between bacterial conjugation and motility is mediated by

2 plasmid-borne regulators.

3 Ainara Luque, Sonia Paytubi, Javier Sánchez-Montejo, Marta Gibert, Carlos

4 Balsalobre* and Cristina Madrid*.

5 Secció de Microbiologia, Virologia i Biotecnologia. Departament de Genètica,

6 Microbiologia i Estadística, Facultat de Biologia, Universitat de Barcelona,

7 Avda. Diagonal 643, 08028 Barcelona (Spain).

$8 \quad$ *Corresponding authors: Carlos Balsalobre and Cristina Madrid. Avda. Diagonal

9 643, 08028 Barcelona, Spain. Phone number: +34 934034622 (Carlos

10 Balsalobre) / +34 934039382 (Cristina Madrid). Fax: +34 93 4034629. E-mail

11 addresses: cbalsalobre@ub.edu (Carlos Balsalobre), cmadrid@ub.edu (Cristina

12 Madrid).

13 Key words: R27, conjugation, motility, TrhRY, HtdA

14 Running title: Crosstalk between plasmid conjugation and motility. 


\section{Summary}

Plasmid conjugation is a major horizontal gene transfer mechanism. The acquisition of a plasmid may cause a perturbation of the cell functions in addition to provide advantageous properties for the recipient cell, such as the gaining of antibiotic resistances. The interplay between plasmid and chromosomal functions has been studied using the IncHI1 plasmid R27. Plasmids of the incompatibility group $\mathrm{HI} 1$, isolated from several Gram-negative pathogens, are associated with the spread of multidrug resistance. Their conjugation is tightly regulated by temperature, being repressed at temperatures within the host $\left(37^{\circ} \mathrm{C}\right)$. In this report, we described that at permissive temperature, when conjugation of plasmid R27 is prompted, a reduction in the motility of the cells is observed. This reduction is mediated by the plasmid-encoded regulators TrhR/TrhY, which together with HtdA form a plasmid -borne regulatory circuit controlling R27 conjugation. TrhR/TrhY, required to induce $\mathrm{R} 27$ conjugation, are responsible of the downregulation of the flagella synthesis and the consequent decrease in motility.

TrhR/TrhYrepress, direct or indirectly, the expression of the specific flagellar sigma subunit FliA and, consequently, the expression of all genes located bellow in the flagellar expression cascade. 
The dissemination of antimicrobial resistance in bacteria has been largely associated to DNA exchange by horizontal gene transfer (HGT) mechanisms. Plasmid conjugation is a major HGT mechanism. The acquisition of a plasmid may result advantageous for the host cell under specific conditions if it confers new phenotypes such as resistance to antibiotics and heavy metals. In other cases, the presence of the plasmid may cause a fitness loss due to perturbation of cell functions.

Plasmids of the incompatibility group HI1, isolated from several species of enterobacteria (Escherichia coli, Salmonella enterica, Klebsiella pneumonia.....) are important vectors of antibiotic resistance genes (Holt et al., 2007; Phan and Wain, 2008, Holt et al., 2011). The conjugative transfer of these plasmids is tightly regulated by temperature, being the conjugation frequency optimal in a temperature range of $22-30^{\circ} \mathrm{C}$ (Maher et al., 1993). This behavior suggest that plasmid transfer is not promoted within the mammal host whereas is induced during the transit of Salmonella in water and/or soil environments. Plasmid R27, encoding resistance to tetracycline, is the prototype of IncHI1 plasmids and it has been intensively studied for over 20 years. Its complete nucleotide sequence is available and the replication and conjugation determinants are well characterized (Taylor et al., 1985; Couturier et al., 1988; Maher et al., 1993; Gabant et al., 1994; Newnham and Taylor, 1994; Sherburne et al., 2000; Lawley et al., 2002, 2003; Alonso et al., 2005). The interplay between the R27 plasmid and the bacterial chromosome has been studied, and functional interactions between regulators from both genetic elements have been 
characterized ( Doyle et al., 2007; Baños et al., 2009; Dillon and Dorman, 2010; Gibert et al., 2014, 2016). Moreover, the impact of the presence of R27 on the global expression profile of Salmonella under some environmental conditions has been described (Paytubi et al., 2014). In previous studies we demonstrated that a regulatory circuit encoded in the R27 conjugative plasmid, formed by HtdA, TrhR and TrhY, tightly controls plasmid conjugation (Gibert et al., 2014). TrhR and TrhY complex, from now named TrhR/Y, are essential to trigger conjugation by stimulating transcriptional expression of the tra genes, whereas HtdA has an overall repressor effect, presumably by acting as an antiactivator of TrhR/Y. We also observed that mutations in the $h t d A$ locus, derepressing R27 conjugation, cause a concomitant decrease in motility (Gibert et al., 2013). Accordingly, the existence of a regulatory crosstalk between expression of conjugative apparatus and bacterial motility was previously anticipated by several authors (Bohlin and Burman, 1977; Maher et al., 1993; Barrios et al., 2006; Reisner et al., 2012; Rösch et al., 2014; Takahashi et al., 2015).

In Escherichia coli, more than 50 genes are involved in the synthesis of a functional flagella, which are classified into three groups according to their temporal expression (Chilcott and Hughes, 2000). The master regulator of flagella synthesis, FlhD-FlhC, is encoded by the class 1 flagellar operon (flhDC). FlhD-FlhC activates the expression of class 2 genes, encoding the inner part of the flagellum, the flagellar sigma factor FliA $\left(\sigma^{28}\right)$ and the anti- $\sigma^{28}$ protein, FlgM.

Class 3 genes are transcribed by a $\sigma^{28}$-RNAP and encode the outer components of the flagellum as well as the chemotaxis proteins. 
81 In this report, we describe an inverse relationship between plasmid conjugation

82 and motility in E.coli. Hence, when conjugation is prompted a reduction in

83 motility is detected. Our studies indicate that TrhR/Y, plasmid-encoded factors

84 that trigger expression of the conjugative apparatus genes, are required for the

85 downregulation of the flagella synthesis and the concomitant decrease in

86 motility. 
Mutations that alter the conjugation frequency of R27 plasmid affect the swimming motility.

To study the crosstalk between conjugation and motility, the plasmid R27 prototype of the IncHI1 plasmids - was used as a model. The swimming ability on TB plates and the conjugation ratio at permissive temperature $\left(25^{\circ} \mathrm{C}\right)$ was determined for $E$. coli strains carrying either the R27 plasmid. The presence of R27 plasmid causes an overall increase in the swimming ability. To establish if motility is affected when the plasmid conjugation is promoted, swimming ability was also monitored for strains carrying R27 or derivatives with mutations for different key regulators of bacterial conjugation: $T r h R / Y$ that are required for induction of R27 conjugation and HtdA that acts as an antiactivator having an overall repressor effect on R27 conjugation. A decrease in the swimming ability of the cells is observed when a derepressed plasmid, drR27 (htdA), is present. In agreement with previous observations (Gibert et al., 2013), absence of htdA (drR27) causes a drastic derepression of R27 conjugation frequency with more than $3 \log _{10}$ increase as compared to wt (Fig. 1A). The presence of drR27 results revealed a correlation between conjugation frequency and motility.. Next, the effect of the trhR mutation (R27 trhR-Tmp), that causes a decrease in the frequency of R27 conjugation as compared to wt (Fig 1A), was tested. . No effect of the trhR mutation was observed in the motility (Fig. 1B and C), which can be a consequence of the very low conjugation frequency $(2.8 \mathrm{E}-5)$ of the R27 plasmid under the conditions used.. Thus, a further decrease in the 
111 conjugation frequency, by introducing the trhR mutation (4.7E-6), would not cause any additive effect on swimming. Consistent with this explanation, the effect of the trhR mutation on motility was evident when using a drR27 plasmid. The trhR mutation ( $\mathrm{drR} 27$ trhR-Tmp) causes a very severe drop in the frequency of conjugation (from 1.1E-1 to 2.3E-5, Fig. 1A) and a clear increase in the motility (Fig. 1B and C), as compared to drR27.

117 Our data indicate that when a cell trigger the conjugative process has the ability to modulate motility. Experiments were performed to determine whether motility can regulate R27 conjugation. The conjugation frequency of R27 and drR27 plasmids was determined in strains deficient in swimming motility by carrying a mutation in the flhDC operon, coding for the master regulator of the flagella biosynthesis cascade. The data obtained (Fig. 1A and B) show that the flhDC mutants are not motile and the conjugation frequency of both R27 and drR27 is not affected by the swimming ability of the donor cell.

TrhR/Y reduces bacterial motility and HtdA counteracts its action.

It can be hypothesized that the crosstalk between R27 conjugation and motility is due to either i) a side effect of the biosynthesis of the conjugational apparatus in the physiology and/or energetic status of the cell, or ii) a consequence of the involvement of specific regulatory circuits controlling both cellular processes, conjugation and motility. The possiblec involvement of the regulatory circuit TrhR/Y-HtdA, which modulates R27 conjugation, in the control of swimming motility was further characterized. The effect of the presence of the regulators TrhR/Y and HtdA on motility, in the absence of the whole R27 conjugative plasmid, was monitored (Figure 2A and 2B). A drop in motility (2.6-fold) was 
135 observed in the presence of plasmid pBAD\#3, carrying the trhR and trhY genes with its own promoter, as compared with the strain carrying the control vector (pBAD18). On the other hand, the presence of plasmid pACYChtdA, carrying the $h t d A$ gene in pACYC184, causes only a slight increase in the motility. This result suggests that TrhR/Y regulators interfere negatively with the chromosomal gene cascade responsible of flagella biosynthesis and motility.

Previously, we described that the regulatory activity of $\operatorname{TrhR} / \mathrm{Y}$ on the control of R27 conjugation is counteracted by the presence of HtdA (Gibert et al., 2014). This interplay TrhR/Y-HtdA is also involved in the motility control since addition of pACYChtdA plasmid restores motility in the strain carrying the pBAD\#3 plasmid (Fig. 2A and 2B).

Further corroboration that the expression of trhR and trhY from the pBAD\#3 plasmid causes a reduction in bacterial motility was obtained using plasmid pBADtrhRY. In plasmid pBADtrhRY, the expression of trhR and trhY genes is under the control of the arabinose inducible $\mathrm{P}_{\mathrm{BAD}}$ promoter. As seen in figure $2 \mathrm{C}$, a decrease in the motility was only observed when trhR trhY expression was induced by the addition of arabinose. Again, the negative effect of TrhR/Y was counteracted by the presence of HtdA. Previously, we described that both, TrhR and TrhY, are required for the transcriptional activation of R27 conjugation genes (Gibert et al., 2014). Similarly, when trhY or trhR were expressed independently no effect on motility was detected, indicating that both, TrhR and TrhY, are required to cause motility reduction. Remarkably, our data also indicate that there is no need of any other R27-encoded factor to reduce motility. 

biosynthesis.

Overall, our results indicated that the $\mathrm{TrhR} / \mathrm{Y}-\mathrm{HtdA}$ regulatory circuit, involved in the transcriptional control of the tra operons and, consequently, R27 conjugation, interferes with swimming motility. Swimming motility can be affected by altering either flagella synthesis or flagella functionality. To discriminate between the two options, flagella synthesis was monitored by direct observation using transmission electron microscopy (Fig. 3A). The data obtained reveals that the expression of $\operatorname{trh} R Y(p B A D \# 3)$, causes a clear the counteractivity described for $\mathrm{HtdA}$, when pACYChtdA plasmid was introduced in cells carrying pBAD\#3, the number of flagella per cell observed increases to similar numbers to those detected in the control strain (no plasmid). Our data clearly demonstrate that the conjugation regulators TrhR/YHtdA affect biosynthesis of flagella. Furthermore, the reduction in the number of flagella when the cell carries pBAD\#3 plasmid correlates with a decrease in the expression of two motility-related genes, fliC and motA (Fig. $3 \mathrm{~B}$ and $\mathrm{C}$ ). Immunodetection of the flagelin FliC, main subunit of the flagellar filament, in a motor subunit of the flagella, was monitored using a lacZ transcriptional fusion. A reduced activity of the motA promoter was detected in the strain that synthesizes TrhR/Y (1.7-fold) (Fig. 3C). Expression of both, fliC and motA, was 
restored when pACYChtdA was introduced into the strains expressing trhR/Y (Fig. 3B and C).

To further corroborate the effect of the regulatory circuit TrhR/Y-HtdA in the transcriptional cascade that regulates flagella biosynthesis, the mRNA levels of several flagellar genes were estimated by qRT-PCR assays (Fig. 3D). The expression of early ( $f l h C$ ), middle ( $f l i A$ and $f l g M$ ) and late (fliC and $\operatorname{mot} A$ ) genes in the flagella biosynthesis cascade was determined in cells expressing $t r h R / Y$ (pBAD\#3) and cells expressing trhR/Y and $h t d A$ (pDAB\#3 and pACYChtdA). FlhC, together with FlhD, is the master regulator required for transcriptional of several middle genes. FliA is the sigma subunit $\left(\sigma^{28}\right)$ that promotes the transcription of late genes and FlgM is an antisigma factor that modulates FliA activity. FliC and MotA are structural proteins of the flagella, encoded in late genes whose expression is FliA dependent. Accordingly with the previous data, the presence of plasmid pBAD\#3 caused a harsh drop in the transcription of the late genes fliC (10-fold), and $\operatorname{mot} A$ (5-fold). Moreover, it causes a significant decrease in the transcription of the middle gene fliA (3.3-fold). In contrast, the expression levels of flgM and flhC were not affected. Again, HtdA counteracts TrhR/Y-mediated repression of fliC, motA and fliA (see expression level in the presence of $p B A D \# 3$ and pACYChtdA). These results indicate that the regulatory circuit TrhR/Y-HtdA modulates cell motility by altering the transcription of some of the middle and late genes required for flagella synthesis. 


\section{Discussion}

Conjugation is a major mechanism for genetic material transfer between bacteria, and represents one of the main processes involved in antibiotic resistance spread. Although acquisition of certain plasmids may provide advantages under specific growth conditions, for instance plasmids carrying genes encoding resistance to antibiotics or heavy metals and metabolic abilities (Frost et al., 2005), the presence of large plasmids can affect certain cellular processes and cause an overall negative effect on the cell fitness. The effect of the presence of conjugative plasmids on bacterial metabolism has been studied in Escherichia coli, Salmonella enterica, Pseudomonas or Bacillus (Wang et al., 2006; Shintani et al., 2009; Paytubi et al., 2014; Rösch et al., 2014; Takahashi et al., 2015; Jiang et al., 2017). Cell motility was described among the cellular processes that can be altered by the presence of conjugative plasmids. It has been reported that both the R plasmid pUM5 in Salmonella enterica and the IncP-7 plasmid pCAR1 from Pseudomonas cause somehow motility inhibition (Bohlin and Burman, 1977; Takahashi et al., 2015). In Bacillus, the presence of pLS20 plasmid also represses motility. Transcriptional studies demonstrated that pSLP20 affects global transcription, being the expression of the motA gene reduced (2-fold) by the presence of this plasmid (Rösch et al., 2014). In E.coli, a negative effect on motility by the presence of IncFII plasmids has been shown (Barrios et al., 2006; Reisner et al., 2012; Jiang et al., 2017). Although the presence of R1drd19 plasmid does not alter the expression of flagella related genes (Barrios et al., 2006), another IncFII plasmid, pHK01, causes a downregulation in the transcriptional expression of chemotaxis and flagellar assembly genes (Jiang et al., 2017). In all the mentioned reports, the effect of 
the presence of conjugative plasmids on motility was determined; however no specific regulators that mediate such plasmid-chromosome crosstalk were identified. It cannot be rule out that these conjugation/motility crosstalks are mediated by a similar mechanisms to that described in this report for R27 plasmid. However, different regulatory factors should be involved since TrhR/Y proteins have been only found encoded among IncHI plasmids (Gibert et al., 2014).

In this work, we show that conjugation of plasmid R27 affects motility in E. coli. The frequency of conjugation of the R27 plasmid is very low and, remarkably, when bacterial conjugation is promoted by the use of the derepressed plasmid drR27 a drastic drop in bacterial motility is detected. One might suggest that sudden biosynthesis of the conjugational apparatus may non-specifically affect cell physiology and/or the energetic status of the cell, which could influence as a side consequence the cellular motility. This is not the case, since we show that the TrhR/Y-HtdA complex, the pivotal regulatory circuit modulating R27 conjugation, is involved in the crosstalk between plasmid conjugation and motility. Ectopical expression of trhR and trhY genes, in the absence of R27 plasmid, causes a clear decrease on motility. TrhR/Y proteins are part of the plasmid-encoded regulatory circuit that controls expression of the tra genes and, consequently, the frequency of conjugation of the plasmid. TrhR and ThrY proteins are both required to induce tra operons expression. HtdA, another component of the regulatory circuit, acts as antiactivator of TrhR/Y having an overall repressor effect (Gibert et al., 2014). TrhR/Y has a dual effect by inducing conjugation and repressing cell motility whereas HtdA counteracts $\mathrm{TrhR} / \mathrm{Y}$ in both cellular processes. Overall, our data indicate that $\mathrm{TrhR} / \mathrm{Y}$ is 
255 required to repress motility and that there is no need of any other R27-encoded

256 factor. This effect is also observed at the level of the expression of flagella-

257 related genes. More than 50 genes are directly involved in motility in E.coli, and are expressed in a temporal-depending manner. The expression of the master regulator, FlhD-FlhC, triggers the expression of the specific sigma subunit $\left(\sigma^{28}\right)$, encoded by fliA, that drives the transcription of the late operons (including fliC and $m o t A$ genes). The expression studies indicate that fliA is repressed in the presence of TrhR/Y and consequently all the genes that are below in the flagellar expression cascade (fliC and motA). Whether TrhR/Y affects directly or indirectly the transcriptional expression of flagellar genes remains elusive. In silico data suggest that TrhY is a cytoplasmatic DNA binding protein and TrhR an inner membrane protein (Gibert et al., 2014). However, the molecular mechanism by which $T r h R / Y$ regulate transcriptional expression of the tra genes is unknown.

Although the effect of large conjugative plasmids on motility was previously described, to our knowledge, this is the first report identifying a specific plasmidencoded factor as responsible of this plasmid-chromosome crosstalk. Further research would be required to gain insight into the exact mechanism underlying this crosstalk. 
274

275

276

277

278

279

280

281

282

283

284

285

286

287

288

289

290

291

292

293

294

295

\section{Experimental procedures}

\section{Bacterial strains, plasmids and growth conditions}

Escherichia coli strains and plasmids used in this study are listed in Table 1. Bacteria were grown in LB (10 g/l NaCl, $10 \mathrm{~g} / \mathrm{l}$ tryptone, $5 \mathrm{~g} / \mathrm{l}$ yeast extract), Penassay broth (1.5 g/l meat extract, $1.5 \mathrm{~g} / \mathrm{l}$ yeast extract, $5 \mathrm{~g} / \mathrm{l}$ peptone, $1 \mathrm{~g} / \mathrm{l}$ glucose, $3.5 \mathrm{~g} / \mathrm{l} \mathrm{NaCl}, 1.32 \mathrm{~g} / \mathrm{KH}_{2} \mathrm{PO}_{4}, 4.82 \mathrm{~g} / \mathrm{K} \mathrm{K}{ }_{2} \mathrm{HP}_{4} \cdot 3 \mathrm{H}_{2} \mathrm{O}$ ), lactose M9 minimal media plates ( $1 \times \mathrm{M} 9$ salts, $0.2 \%$ lactose, $10 \mu \mathrm{M}$ thiamine and $1.5 \%$ agar), TB plates (10 g/l tryptone, $5 \mathrm{~g} / \mathrm{l} \mathrm{NaCl}, 2.5 \mathrm{~g} / \mathrm{l}$ agar) and LB agar plates (LB plus $15 \mathrm{~g} / \mathrm{l}$ agar), as indicated. When needed, antibiotics were added at the following concentrations: tetracycline (Tc) $15 \mu \mathrm{g} / \mathrm{ml}$, chloramphenicol (Cm) 20 $\mu \mathrm{g} / \mathrm{ml}$, ampicillin (Amp) $50 \mu \mathrm{g} / \mathrm{ml}$ or kanamycin $(\mathrm{Km}) 50 \mu \mathrm{g} / \mathrm{ml}$. For induction assays, arabinose was added at concentrations of $0.2 \%(\mathrm{w} / \mathrm{v})$.

To construct the AML strain, carrying a transcriptional fusion of the motA promoter with the lacZ gene, the motA promoter region was PCR amplified using the primer pair MotA-F-Eco and MotA-R-Bam. Next, the PCR-amplified fragment was cloned into EcoRI-BamHI sites of pRS551 (Simons et al., 1987), resulting in plasmid pRS-motA. The motA::lacZ fusion was transferred to the attB lambda site in the chromosome of the AAG1 strain, using previously described protocols (Simons et al., 1987). Controls to confirm single gene fusion insertion in the attB locus were performed as previously described (Powell et al., 1994).

\section{Plasmid conjugation}


296 For conjugation experiments, cultures of donor (AAG1 derivatives, lac-) and

297

298

299

300

301

302

303

304

305

306

307

308

309

310

311

312

313

314

315

316

317

recipient (MG1655, $\mathrm{lac}^{+}$) strains were grown in Penassay broth (PB) supplemented with the required antibiotics at $25^{\circ} \mathrm{C}$ in static conditions for $16 \mathrm{~h}$.

Cells were washed with PB to eliminate the antibiotics. Aliquots of recipient strain suspension $(50 \mu \mathrm{l})$ and donor strain suspension $(25 \mu \mathrm{l})$ were added to a filter placed on a LB agar plate. After $2 \mathrm{~h}$ incubation at $25^{\circ} \mathrm{C}$, the filter was placed in a tube and washed with $1.5 \mathrm{ml}$ of Penassay broth to free the bacteria from the filter. Serial dilutions were plated in LB agar plates supplemented with IPTG $(0.5 \mathrm{mM})$ and $\mathrm{Xgal}(40 \mu \mathrm{g} / \mathrm{ml})$ or lactose $\mathrm{M} 9$ minimal media plates to discriminate between donor ( $\left(a c^{-}\right)$and transconjugant $\left(\mathrm{lac}^{+}\right)$cells, as previously described (Gibert et al., 2013). When MG1655 flhD::Cm was used as a donor strain, strain AML was used as recipient, and transconjugants were selected in LB plates supplemented with tetracycline and kanamycin. The mating frequency was calculated as the number of transconjugants per donor cell. We show the average of three independent experiments plotted with standard deviation.

\section{Motility Assays}

The motility phenotype was analyzed on TB plates. Overnight LB bacterial cultures at $25^{\circ} \mathrm{C}$ were spotted $(5 \mu \mathrm{l})$ on the center of the plates and incubated at $25^{\circ} \mathrm{C}$. The colony diameter was measured and plotted, and standard deviations were calculated. The data are representative of two independent experiments with four replica plates for each strain.

\section{Transmission electron microscopy}


318 Bacterial strains used for flagella visualization were grown in LB at $25^{\circ} \mathrm{C}$ in static conditions for 16 hours. Bacterial cells were centrifuged $(1,500 \times g$ for 5 min) and resuspended in filtered Ringer $1 / 4$ solution. Cu-Carbon grid (CF200-Cu Carbon Film On 200 Mesh Copper Grids, Electron Microscopy Sciences) was soaked for $60 \mathrm{sec}$ on a $5 \mu \mathrm{l}$ drop of each strain resuspension, washed three times with water for $20 \mathrm{sec}$ and stained for $60 \mathrm{sec}$ using a $2 \%(\mathrm{w} / \mathrm{v})$ uranyl acetate solution (Polysciences). Once stained, the grids were dried for at least $24 \mathrm{~h}$ before visualization under a JEOL JEM1010 transmission electron microscope. Images were obtained using the software analySIS (Soft Imaging System $\mathrm{GmbH}$, Münster, Germany). Each sample was observed for at least 100 cells.

\section{Total protein extracts}

To obtain total protein extracts, bacterial strains were grown at $25^{\circ} \mathrm{C}$ in shacking conditions for 16 hours and total protein was precipitated.

Trichloracetic acid (TCA) was added to bacterial cultures at $20 \%$ final concentration, mixtures were incubated for $1 \mathrm{~h}$ on ice and centrifuged $(14,000$ $\left.\mathrm{xg}, 4^{\circ} \mathrm{C}\right)$. Precipitates were washed with $80 \%$ cold acetone, air dried and resuspended in protein sample buffer. Final volume was adjusted to the original $\mathrm{OD}_{600}$ of each culture.

\section{Gel electrophoresis and Western blot}

Protein samples were analyzed by SDS-PAGE and immunoblotted by western blot upon transfer of proteins to PDVF membranes. Western blot analysis was performed with polyclonal antibodies raised against $E$. coli FliC protein [1:2000] 
341 (Westerlund-Wikström et al., 1997). Horseradish peroxidase-conjugated goat anti-rabbit IgG [1:10000] (Sigma) was used as secondary antibody. ECL Prime Western Blotting detection reagent (GE Healthcare) was used to immunodetect the transferred proteins. Visualization and analysis of the detected bands was performed using Molecular Imager ChemiDoc XRS System and Quantity One software (Bio Rad).

\section{$\beta$-galactosidase assay}

$\beta$-galactosidase assays were performed as described by Miller (Miller, 1992) using cultures grown in $\mathrm{LB}$ at $25^{\circ} \mathrm{C}$ until an $\mathrm{OD}_{600}$ of 2.0. Data are mean values of duplicate determinations in at least three independent experiments plotted with standard deviations.

\section{Total RNA isolation}

Total RNA was isolated from three independent cultures of the AAG1 derivative strains grown at $25^{\circ} \mathrm{C}$ in LB until an $\mathrm{OD}_{600}$ of 2.0 , using a SV Total RNA Isolation System (Promega) according to manufacturer's directions. The purity and quality of the purified RNA was tested by Bioanalyzer 2100 (Agilent Technologies).

\section{qRT-PCR}

359 The expression level of $f l i C$, fliA, motA, flhC and flgM genes was determined by real-time quantitative reverse transcription-PCR (qRT-PCR). Briefly, $1 \mu \mathrm{g}$ of total RNA was reverse transcribed to generate cDNA using the "High-capacity cDNA Reverse Transcription kit" (Applied Biosystems) as recommended by the 
manufacturer. As a control, samples in which reverse transcriptase was omitted

364 from the reaction mixture were used. Real-time PCR using "Power SYBR Green

365 PCR Master Mix kit" (Applied Biosystems) was carried out on the StepOne

366 Real-Time PCR System Thermal Cycling Block (Applied Biosystems).

367 Oligonucleotides to PCR amplification of the genes of interest were designed

368 using Primer3 online tool (Rozen and Skaletsky, 2000) provided by the

369 Whitehead institute (http://bioinfo.ut.ee/primer3/) (Table 2). The relative amount

370 of target cDNA was normalized using gapdh gene as an internal reference

371 standard. The relative expression was expressed as fold change using the

372 expression level in wild type strain set arbitrarily to 1.0. 


\section{Acknowledgements}

374 This work was supported by grants from the Spanish Ministry of Science and 375 Innovation (AGL2013-45339-R) and the Generalitat de Catalunya (2017 SGR 376 499).

\section{Conflict of interest}

378 None to declare.

\section{References}

380

381

382

383

384

Aberg, A., Shingler, V., and Balsalobre, C. (2008) Regulation of the fimB promoter: a case of differential regulation by ppGpp and DksA in vivo. Mol. Microbiol. 67: 1223-41.

Alonso, G., Baptista, K., Ngo, T., and Taylor, D.E. (2005) Transcriptional organization of the temperature-sensitive transfer system from the IncHI1 plasmid R27. Microbiology 151: 3563-3573.

Baños, R.C., Vivero, A., Aznar, S., García, J., Pons, M., Madrid, C., and Juárez, A. (2009) Differential regulation of horizontally acquired and core genome genes by the bacterial modulator H-NS. PLoS Genet. 5: e1000513.

Barrios, A.F.G., Zuo, R., Ren, D., and Wood, T.K. (2006) Hha, YbaJ, and OmpA regulate Escherichia coli K12 biofilm formation and conjugation plasmids abolish motility. Biotechnol. Bioeng. 93: 188-200.

Bohlin, T. and Burman, L.G. (1977) Influence on motility of Escherichia coli and Salmonella typhimurium by a naturally occurring conjugative plasmid. J. Bacteriol. 130: 604-9. 
Chilcott, G.S. and Hughes, K.T. (2000) Coupling of flagellar gene expression to flagellar assembly in Salmonella enterica serovar typhimurium and Escherichia coli. Microbiol. Mol. Biol. Rev. 64: 694-708.

Couturier, M., Bex, F., Bergquist, P.L., and Maas, W.K. (1988) Identification and classification of bacterial plasmids. Microbiol. Rev. 52: 375-95.

Dillon, S.C. and Dorman, C.J. (2010) Bacterial nucleoid-associated proteins, nucleoid structure and gene expression. Nat. Rev. Microbiol. 8: 185-95.

Doyle, M., Fookes, M., Ivens, A., Mangan, M.W., Wain, J., and Dorman, C.J. (2007) An H-NS-like stealth protein aids horizontal DNA transmission in bacteria. Science 315: 251-2.

Fontaine, F., Stewart, E.J., Lindner, A.B., and Taddei, F. (2008) Mutations in two global regulators lower individual mortality in Escherichia coli. Mol. Microbiol. 67: 2-14.

Frost, L.S., Leplae, R., Summers, A.O., and Toussaint, A. (2005) Mobile genetic elements: the agents of open source evolution. Nat. Rev. Microbiol. 3: 722732.

Gabant, P., Chahdi, A.O., and Couturier, M. (1994) Nucleotide sequence and replication characteristics of RepHI1B: a replicon specific to the IncHI1 plasmids. Plasmid 31: 111-120.

Gibert, M., Juárez, A., Madrid, C., and Balsalobre, C. (2013) New insights in the role of $\mathrm{HtdA}$ in the regulation of R27 conjugation. Plasmid 70: 61-8.

Gibert, M., Juárez, A., Zechner, E.L., Madrid, C., and Balsalobre, C. (2014) TrhR, TrhY and HtdA, a novel regulatory circuit that modulates conjugation of the IncHI plasmids. Mol. Microbiol. 94: 1146-1161. 
419 Gibert, M., Paytubi, S., Beltrán, S., Juárez, A., Balsalobre, C., and Madrid, C.

420

421

422

423

424

425 (2016) Growth phase-dependent control of R27 conjugation is mediated by the interplay between the plasmid-encoded regulatory circuit TrhR/TrhY$\mathrm{HtdA}$ and the cAMP regulon. Environ. Microbiol. 18: 5277-5287.

Grindley, N.D., Grindley, J.N., and Anderson, E.S. (1972) R factor compatibility groups. Mol. Gen. Genet. 119: 287-97.

Guyer, M.S., Reed, R.R., Steitz, J.A., and Low, K.B. (1981) Identification of a sex-factor-affinity site in E. coli as gamma delta. Cold Spring Harb. Symp. Quant. Biol. 45 Pt 1: 135-40.

Guzman, L., Belin, D., Carson, M., and Beckwith, J. (1995) Tight regulation, modulation, and high-level expression by vectors containing the arabinose PBAD promoter. J. Bacteriol. 177: 4121-4130.

Holt, K.E., Phan, M.D., Baker, S., Duy, P.T., Nga, T.V.T., Nair, S., et al. (2011) Emergence of a globally dominant IncHI1 plasmid type associated with multiple drug resistant typhoid. PLoS Negl. Trop. Dis. 5: e1245.

Holt, K.E., Thomson, N.R., Wain, J., Phan, M.D., Nair, S., Hasan, R., et al. (2007) Multidrug-Resistant Salmonella enterica serovar Paratyphi A harbors IncHI1 plasmids similar to those found in serovar Typhi. J. Bacteriol. 189: 4257-4264.

Jiang, X., Liu, X., Law, C.O.K., Wang, Y., Lo, W.U., Weng, X., et al. (2017) The CTX-M-14 plasmid pHK01 encodes novel small RNAs and influences host growth and motility. FEMS Microbiol. Ecol. 93:fix 090.

Lawley, T.D., Gilmour, M.W., Gunton, J.E., Standeven, L.J., and Taylor, D.E. (2002) Functional and mutational analysis of conjugative transfer region 1 
(Tra1) from the IncHI1 plasmid R27. J. Bacteriol. 184: 2173-80.

Lawley, T.D., Gilmour, M.W., Gunton, J.E., Tracz, D.M., and Taylor, D.E. (2003) Functional and mutational analysis of conjugative transfer region 2 (Tra2) from the IncHI1 plasmid R27. J. Bacteriol. 185: 581-91.

Maher, D., Sherburne, R., and Taylor, D.E. (1993) H-pilus assembly kinetics determined by electron microscopy. J. Bacteriol. 175: 2175-83.

Miller, J.H. (1992) A short course in bacterial genetics : a laboratory manual and handbook for Escherichia coli and related bacteria Cold Spring Harbor Laboratory Press.

Newnham, P.J. and Taylor, D.E. (1994) Molecular analysis of RepHI1A, a minimal replicon of the IncHI1 plasmid R27. Mol. Microbiol. 11: 757-68.

Paytubi, S., Aznar, S., Madrid, C., Balsalobre, C., Dillon, S.C., Dorman, C.J., and Juárez, A. (2014) A novel role for antibiotic resistance plasmids in facilitating Salmonella adaptation to non-host environments. Environ. Microbiol. 16: 950-62.

Phan, M.-D. and Wain, J. (2008) IncHI plasmids, a dynamic link between resistance and pathogenicity. J. Infect. Dev. Ctries. 2: 272-8.

Powell, B.S., Rivas, M.P., Court, D.L., Nakamura, Y., and Turnbough, C.L. (1994) Rapid confirmation of single copy lambda prophage integration by PCR. Nucleic Acids Res. 22: 5765-6.

Reisner, A., Wolinski, H., and Zechner, E.L. (2012) In situ monitoring of IncF plasmid transfer on semi-solid agar surfaces reveals a limited invasion of plasmids in recipient colonies. Plasmid 67: 155-61.

Rösch, T.C., Golman, W., Hucklesby, L., Gonzalez-Pastor, J.E., and 
Graumann, P.L. (2014) The presence of conjugative plasmid pLS20 affects global transcription of Its Bacillus subtilis host and confers beneficial stress resistance to cells. Appl. Environ. Microbiol. 80: 1349-58.

Rose, R.E. (1988) The nucleotide sequence of pACYC184. Nucleic Acids Res. 16: 355 .

Rozen, S. and Skaletsky, H. (2000) Primer3 on the WWW for general users and for biologist programmers. Methods Mol. Biol. 132: 365-86.

Sherburne, C.K., Lawley, T.D., Gilmour, M.W., Blattner, F.R., Burland, V., Grotbeck, E., et al. (2000) The complete DNA sequence and analysis of $\mathrm{R} 27$, a large IncHI plasmid from Salmonella typhi that is temperature sensitive for transfer. Nucleic Acids Res. 28: 2177-86.

Shintani, M., Takahashi, Y., Tokumaru, H., Kadota, K., Hara, H., Miyakoshi, M., et al. (2009) Response of the Pseudomonas host chromosomal transcriptome to carriage of the IncP-7 plasmid pCAR1. Environ. Microbiol. 12: 1413-26.

Simons, R.W., Houman, F., and Kleckner, N. (1987a) Improved single and multicopy lac-based cloning vectors for protein and operon fusions. Gene 53: 85-96.

Simons, R.W., Houman, F., and Kleckner, N. (1987b) Improved single and multicopy lac-based cloning vectors for protein and operon fusions. Gene 53: 85-96.

Takahashi, Y., Shintani, M., Takase, N., Kazo, Y., Kawamura, F., Hara, H., et al. (2015) Modulation of primary cell function of host Pseudomonas bacteria by the conjugative plasmid pCAR1. Environ. Microbiol. 17: 134- 

55.

492

493

494

495

496

497

498

499

500

501

502

503

504

505

506

507

508

509

510

\section{1}

Taylor, D.E., Brose, E.C., Kwan, S., and Yan, W. (1985) Mapping of transfer regions within incompatibility group HI plasmid R27. J. Bacteriol. 162: 1221-6.

Wang, Z., Xiang, L., Shao, J., Węgrzyn, A., and Węgrzyn, G. (2006) Effects of the presence of ColE1 plasmid DNA in Escherichia coli on the host cell metabolism. Microb. Cell Fact. 5: 34.

Westerlund-Wikström, B., Tanskanen, J., Virkola, R., Hacker, J., Lindberg, M., Skurnik, M., and Korhonen, T.K. (1997) Functional expression of adhesive peptides as fusions to Escherichia coli flagellin. Protein Eng. 10: 1319-26.

2

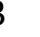




\section{$511 \quad$ Figure legends}

512 Figure 1. High conjugation frequency correlates with a reduction in the ability to swim. A) Conjugation frequencies of R27 plasmid and its derivatives using AAG1 or MG1655 fIhD::Cm as the donor and MG1655 or AML respectively as the recipient strains. B) Swimming motility on TB plates after 15 hours incubation at $25^{\circ} \mathrm{C}$ for strains carrying the indicated plasmids (n.p no plasmid)) C) The diameter of the perimeter of the bacterial growth in (B) was measured (dashed lines), and averages and standard deviations were plotted.

Figure 2. Effect of TrhRY and HtdA on the swimming phenotype. A) Motility on TB plates after 15 hours incubation at $25^{\circ} \mathrm{C}$ of strain MG1655 carrying indicated plasmids. B) The diameter of the perimeter of bacterial growth in (A) was measured (dashed lines), and averages and standard deviations were plotted. C) Motility on TB plates after 20 hours incubation at $25^{\circ} \mathrm{C}$ of strain MG1655 carrying plasmids indicated in the X-axis, in the presence and absence of $0.2 \%$ arabinose.

Figure 3. Effect of TrhR/Y and HtdA on the flagella biosynthesis. A)

Visualization of MG1655 cells carrying the plasmids indicated (n.p, no plasmid) using transmission electron microscopy. B) Immunodetection of FliC on total protein extracts of strain MG1655 carrying the indicated plasmids (n.p, no plasmid). The relative amount of FliC was compared to that corresponding to the control without any plasmid (n.p.). OmpA immunodetection was used as a control. C) Transcriptional expression of the promoter sequence of $\operatorname{mot} A$ in the absence or presence of regulators TrhRY (pBAD\#3) or HtdA (pACYChtdA)

534 monitored as $\beta$-galactosidase activity (Miller units). Data are mean values of 
535 duplicate determinations of at least three independent cultures plotted with

536 standard deviations. D) Expression level of fliC, fliA, $\operatorname{mot} A$, flhC and flgM

537 determined by qRT-PCR in the absence (pBAD18) or the presence of TrhRY

538 (pBAD\#3) or TrhR/Y and HtdA (pBAD\#3 pACYChtdA) regulators. 
539 Table 1. Bacterial strains and plasmids

540

\begin{tabular}{|c|c|c|}
\hline Strain & Genotype & Reference \\
\hline MG1655 & $\mathrm{F}^{-}$, ilvG, rph1 & (Guyer et al., 1981) \\
\hline AAG1 & MG1665 $\Delta / a c$ & (Aberg et al., 2008) \\
\hline MG1655 flhD::Cm & flhD::Cm, $\mathrm{Cm}^{\mathrm{R}}$ & (Fontaine et al., 2008) \\
\hline AML & AAG1 motA::/acZR & This work \\
\hline Plasmids & Genotype & Reference \\
\hline $\mathrm{R} 27$ & IncHI1, Tc ${ }^{R}$ & (Grindley et al., 1972) \\
\hline drR27 & R27 htdA::IS10, Tc ${ }^{R}$ & (Gibert et al., 2013) \\
\hline R27trhR-Tmp & R27 trhR::Tmp ${ }^{R}$ & (Gibert et al., 2014) \\
\hline drR27trhR-Tmp & drR27 trhR::Tmp R & \\
\hline pRS551 & $\begin{array}{l}\text { lac based transcriptional fusion } \\
\text { vector, } A m p^{R} \mathrm{Km}^{R}\end{array}$ & (Simons et al., 1987) \\
\hline pRS-motA & $\mathrm{P}_{\text {motA }}$ cloned into pRS551 & This work \\
\hline pACYC184 & Cloning vector, $\mathrm{Cm}^{\mathrm{R}}, \mathrm{Tc}^{\mathrm{R}}$ & (Rose, 1988) \\
\hline pACYChtdA & pACYC184 + htdA & (Gibert et al., 2014) \\
\hline pDAB18 & Cloning vector, $\mathrm{Amp}^{\mathrm{R}}$ & (Guzman et al., 1995) \\
\hline pBAD\#3 & $\begin{array}{l}\text { pBAD18 carrying an EcoRI } \\
\text { fragment of R27 (trhRtrhY) }\end{array}$ & (Gibert et al., 2014) \\
\hline pBADtrhR & pBAD18 + trhR & (Gibert et al., 2014) \\
\hline
\end{tabular}


Table 2. Primers used in this work

\begin{tabular}{|l|l|}
\hline Primer & Sequence 5' $\rightarrow \mathbf{3}^{\prime}$ \\
\hline FliC_F & GCTATCGCATCTGTAGACAA \\
\hline FliC_R & GTAGTGGTGTTGTTCAGGTT \\
\hline FliA_F & CTGAGGTAGCGGAACGTTTA \\
\hline FliA_R & AGAGCTGGCTGTTATTGGTG \\
\hline motA_F & ATGCAGTGCGTCAAAGTCAC \\
\hline motA_R & GCTGGAATAGAGCGTTTGC \\
\hline flhC_F & AGCTTATGTCAACCGCCATC \\
\hline flhC_R & GTGGGATAATATCGGCAGGA \\
\hline flgM_F & CAAGCAAAACTGATGCAACC \\
\hline flgM_R & ACGAATCGCCAGTTTTAACG \\
\hline zwf_F & CACGCGTAGTCATGGAGAAA \\
\hline Zwf_R & CCAAGATAGTGGTCGATACG \\
\hline motA-F-Eco & CCGGAATTCCCTTGAACAGYGCCCACAAG \\
\hline motA-R-Bam & CGCGGATCCACCGCCGAAACTGTACCGA \\
\hline
\end{tabular}



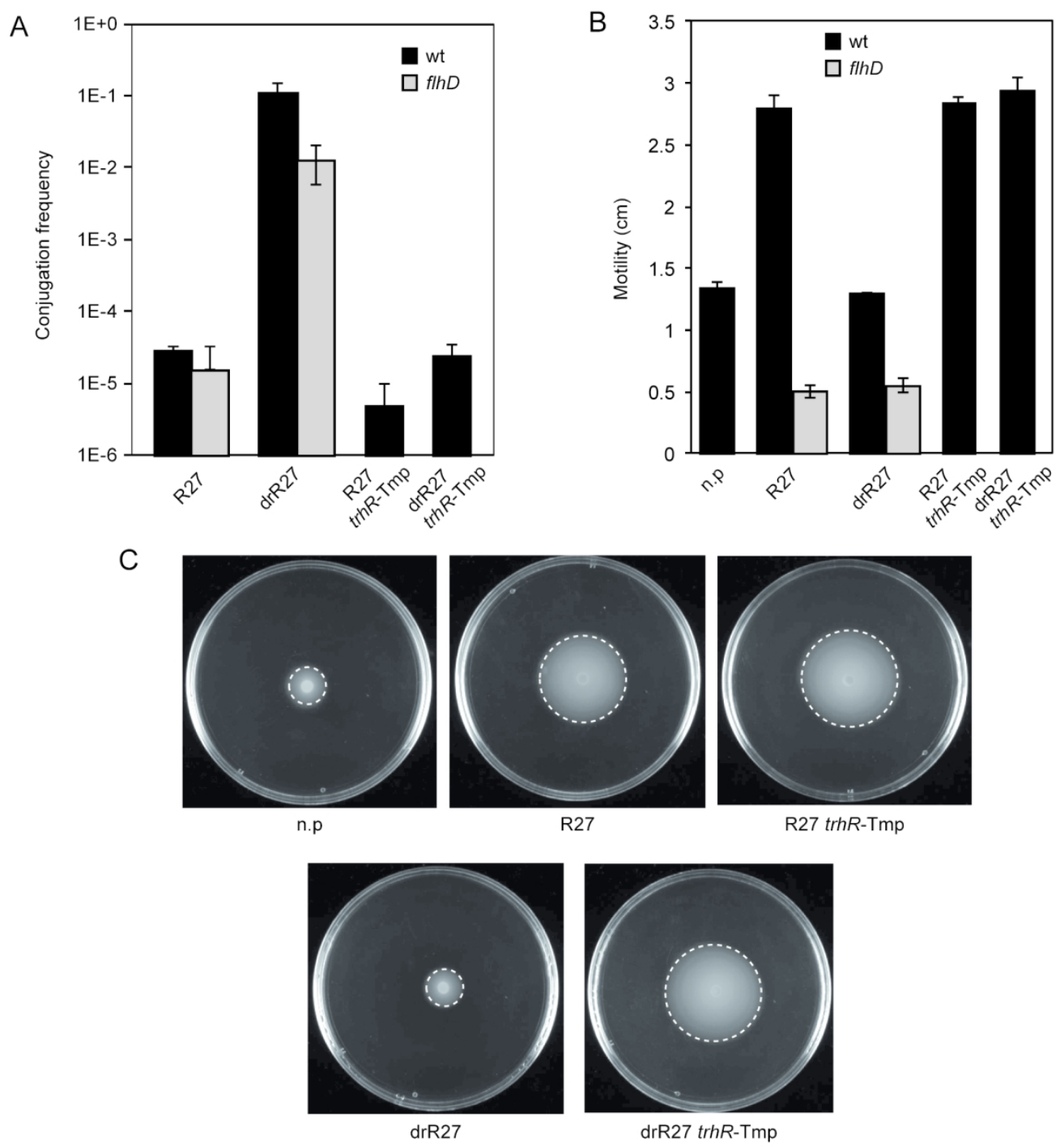

Figure 1

Figure 1. High conjugation frequency correlates with a reduction in the ability to swim 

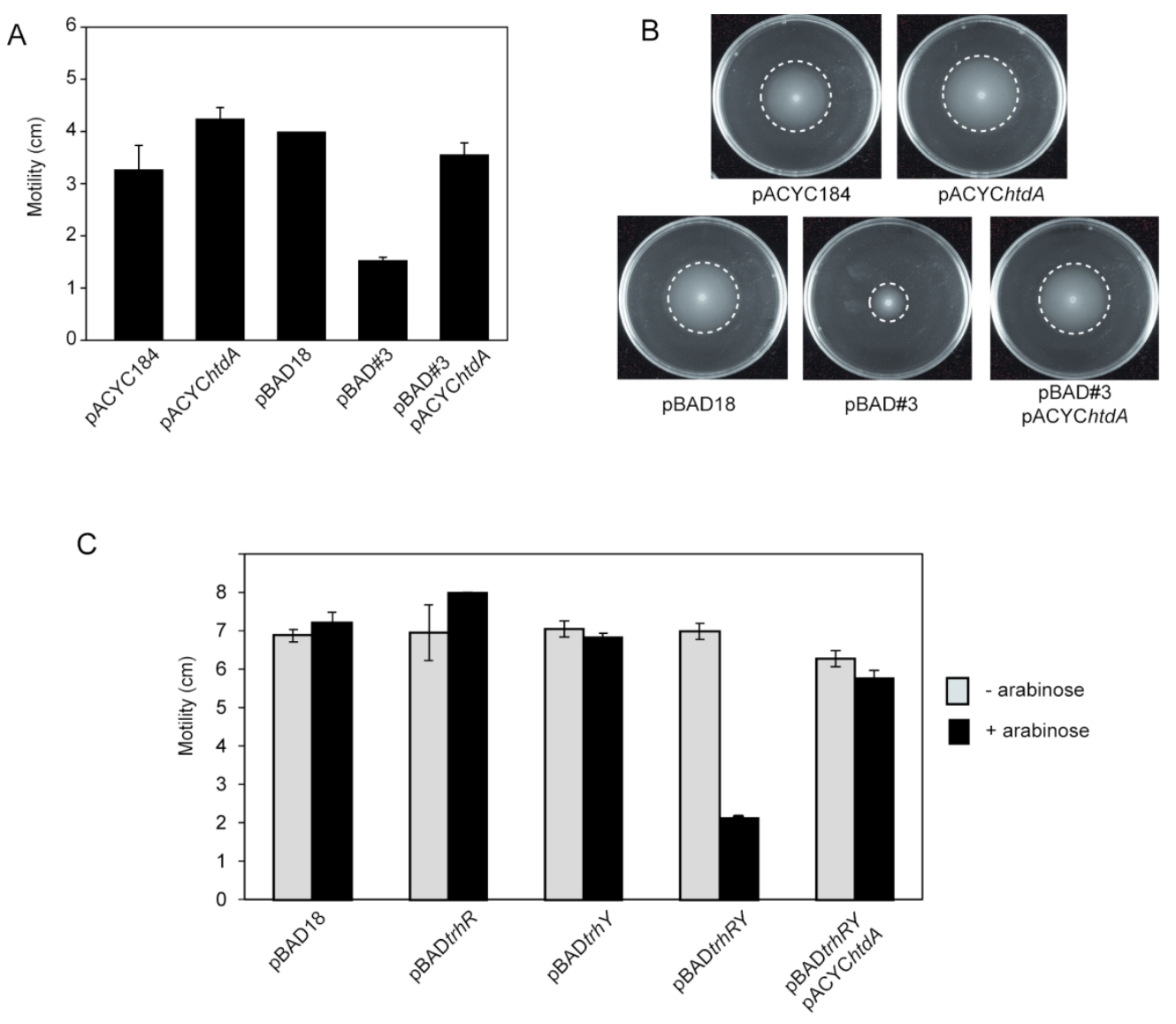

Figure 2

Figure 2. Effect of TrhRY and HtdA on the swimming phenotype 
A
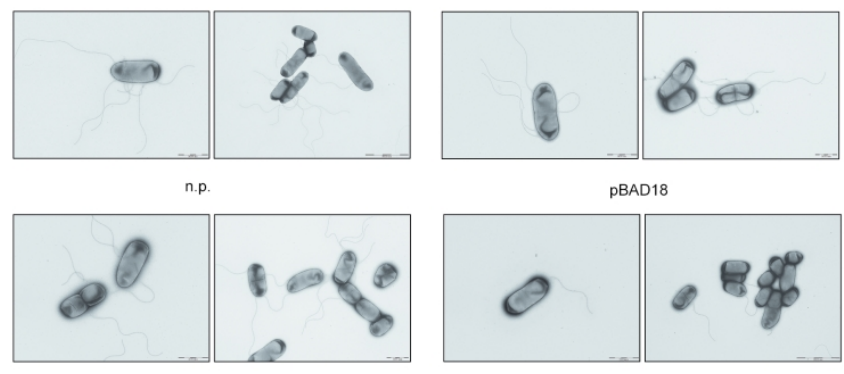

pACYC184

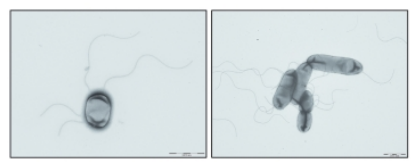

pACYChtdA

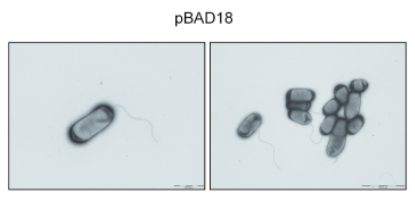

pBAD\#3

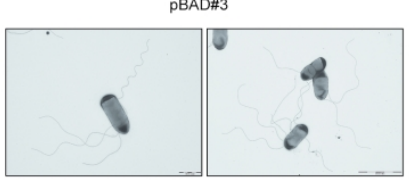

PBAD\#3 pACYChtdA

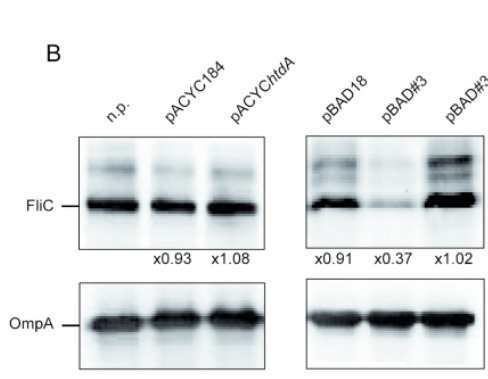

C

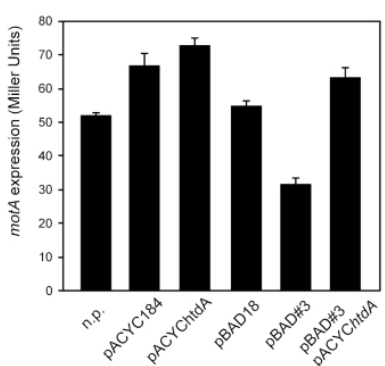

D

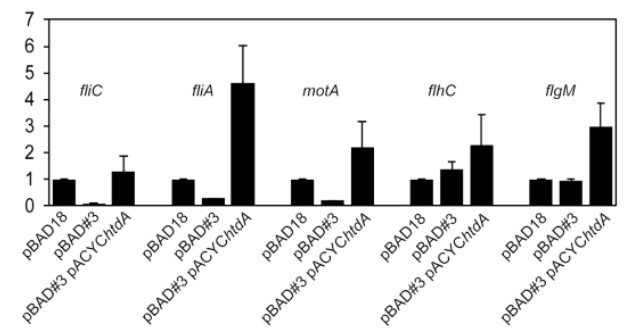

Figure 3

Figure 3. Effect of TrhR/Y and HtdA on the flagella biosynthesis 